\title{
A synthesis method for cobalt doped carbon aerogels with high surface area and their hydrogen storage properties
}

\author{
H.Y. Tian ${ }^{1,2 *}$, C.E. Buckley ${ }^{1,2 *}$, D.A. Sheppard ${ }^{1}$, M. Paskevicius ${ }^{1}$, N. Hanna ${ }^{3}$ \\ ${ }^{1}$ Department of Imaging and Applied Physics, Curtin University of Technology, GPO \\ Box U 1987, Perth 6845, WA, Australia. \\ ${ }^{2}$ CSIRO National Hydrogen Materials Alliance, CSIRO Energy Centre, 10 Murray \\ Dwyer Circuit, Steel River Estate, Mayfield West, NSW 2304, Australia. \\ ${ }^{3}$ CSIRO Process Science and Engineering, Waterford, WA, Australia.
}

\begin{abstract}
Carbon aerogels doped with nanoscaled Co particles were prepared by first coating activated carbon aerogels using a wet-thin layer coating process. The resulting metal doped carbon aerogels had a higher surface area $\left(\sim 1667 \mathrm{~m}^{2} \mathrm{~g}^{-1}\right)$ and larger micropore volume $\left(\sim 0.6 \mathrm{~cm}^{3} \mathrm{~g}^{-1}\right)$ than metal doped carbon aerogels synthesised using other methods suggesting their usefulness in catalytic applications. The hydrogen adsorption behaviour of cobalt-doped carbon aerogel was evaluated, displaying a high $\sim 4.38$ wt. $\% \mathrm{H}_{2}$ uptake under $4.6 \mathrm{MPa}$ at $-196^{\circ} \mathrm{C}$. The hydrogen uptake capacity with respect to unit surface area was greater than for pure carbon aerogel and resulted in $\sim 1.3 \mathrm{H}_{2}$ (wt. \%) per $500 \mathrm{~m}^{2} \mathrm{~g}^{-1}$. However, the total hydrogen uptake was slightly reduced as compared to pure carbon aerogel due to a small reduction in surface area associated with cobalt doping. The improved adsorption per unit surface area suggests that there is a stronger interaction between the hydrogen molecules and the cobalt doped carbon aerogel than for pure carbon aerogel.
\end{abstract}

*Corresponding author. Fax: 61-8-92662377. E-mail address: H.Tian@curtin.edu.au (H.Y. Tian) and C.Buckley@ curtin.edu.au (C.E.Buckley) 


\section{Introduction}

Carbon aerogels (CAs) have drawn much attention as effective physisorbents and as components in other energy storage applications such as fuel cells because of their abundant pores and favorable electrochemical properties [1 -4]. Recently, one area of significant interest involves the incorporation of metal species into the CA framework with the goal of modifying its structure and catalytic activity [5]. Metal-doped CAs have shown an increased enthalpy of adsorption for hydrogen [2], and as such have been investigated as a hydrogen storage medium. Previously, an ion exchange method has been used to uniformly incorporate metal ions into a CA matrix. This process involves producing a potassium-doped hydrogel via the base catalysed polymerization of an aqueous solution of formaldehyde and a potassium salt. The potassium ions can then be replaced with the desired metal ion via ion exchange and the metal-doped hydrogels can then be pyrolysed to generate a metal-doped CA [6]. This metal doping method, however, lacks the crucial step of activation that results in the high surface area and microporosity typical of CAs. For example, it has been shown that for pure CAs, activation leads to a $53 \%$ increase in surface area (from $1005 \mathrm{~cm}^{-1} \mathrm{~g}^{-1}$ to 1539 $\mathrm{cm}^{-2} \mathrm{~g}^{-1}$ ) and a $31 \%$ increase in micropore volume (from $0.51 \mathrm{~cm}^{3} \mathrm{~g}^{-1}$ to $0.71 \mathrm{~cm}^{3} \mathrm{~g}^{-1}$ ) [7]. The activation process for metal doped CAs is avoided as the high temperature that is required results in significant growth of the metal particles as well as deposition of a graphitic coating on the metal particles, hindering access for gaseous molecules such as hydrogen. Additionally, it is our experience that the presence of cobalt during activation greatly accelerates carbon loss, in accord with previous findings [8]. 
Our goal herein was to develop a new method that would allow us to maintain the high surface area and microporosity of pure CA, whilst also incorporating a uniform distribution of metallic cobalt particles.

\section{Experimental}

In this study, CAs were prepared from resorcinol (R) (99.0\%), furfural (F) (99.0\%), and Hexamethylenetetramine (HMTA) (99.0\%), that were all obtained from Sigma Aldrich Pty. Ltd., Australia. The bare CA synthesis procedure is detailed in Ref. [7]. A $0.1 \mathrm{M}$ cobalt solution was prepared from cobalt (II) acetate tetrahydrate $\left(\mathrm{CH}_{3} \mathrm{COO}\right)_{2} \mathrm{Co} \cdot 4 \mathrm{H}_{2} \mathrm{O}$, reagent grade, Sigma Aldrich) in deionised water for the coating process. The solution of cobalt acetate was then added to the CA dropwise and the resultant cobalt slurry was centrifuged for $1 \mathrm{hr}$ to load the metal ions into the pores of the CA. The cobalt acetate solution was then allowed to evaporate from the $\mathrm{CA}$ and the cobalt acetate now contained within the CA was decomposed at $600^{\circ} \mathrm{C}$ in flowing nitrogen $\left(\mathrm{N}_{2}\right.$ flow rate $\left.=1.5 \mathrm{~L} / \mathrm{min}\right)$. Given the quantity and concentration of the cobalt acetate solution and the fact that it was evaporated from the CA means that the maximum possible cobalt loading within the CA is $7.2 \mathrm{wt} \%$. Following this cobalt acetate decomposition step, the sample was reduced under a gas flow $\left(10 \% \mathrm{H}_{2}\right.$ and $90 \%$ Ar) at $500^{\circ} \mathrm{C}$ for $2 \mathrm{hr}$. In summary, the synthesis method involves: (1) synthesis of the organic gel; (2) the carbonisation of the organic gel under flowing $\mathrm{N}_{2}$ : (3) activation in flowing $\mathrm{CO}_{2}$ (4) the loading of the $\mathrm{CA}$ with cobalt acetate solution: (5) the decomposition of cobalt acetate to cobalt oxide under flowing $\mathrm{N}_{2}$ and: (6) the reduction of cobalt oxide to cobalt under a flowing $\mathrm{H}_{2} /$ Ar mixture. The pure carbon aerogel and the Co-doped CA will be referred to as CA and CA-Co-I, respectively. For comparison, a further Co doped sample was also prepared by the ion-exchange method [6], which is referred to as CA-Co-II. The CA-Co-II sample was not activated 
under flowing $\mathrm{CO}_{2}$, and was only carbonised at $900^{\circ} \mathrm{C}$ under a flowing nitrogen atmosphere $\left(\mathrm{N}_{2}\right.$ flow rate $\left.=1.5 \mathrm{~L} / \mathrm{min}\right)$.

The decomposition of cobalt acetate within the CA was verified to reach completion via TGA/DTA analysis performed on an SDT V3.0F (2960) instrument under flowing nitrogen. The X-ray diffraction (XRD) patterns were obtained from a Bruker D8 Advance to identify the crystalline phases present, using a wave-length, $\lambda=0.15406$ $\mathrm{nm}\left(\mathrm{Cu} K_{\alpha}\right)$ with a Lynx Eye area detector for rapid data collection. TEM was performed with a JOEL-2000FX microscope operating at $200 \mathrm{kV}$. TEM Samples were deposited onto carbon coated copper grids from ethanol suspensions. After synthesis was complete all samples were evacuated at $250^{\circ} \mathrm{C}$ overnight, and the hydrogen uptake was evaluated over the pressure range $0-5 \mathrm{MPa}$ at $-196^{\circ} \mathrm{C}$ and room temperature (RT). Hydrogen sorption experiments were performed in a Sieverts manometric apparatus. For ambient temperatures, the Hemmes equation of state [9] was used following the method of McLennan and Gray [10] to account for the compressibility of hydrogen. For $T=-196^{\circ} \mathrm{C}$, the NIST Standard Reference Database Number 69 was used [11].

\section{Results and discussion}

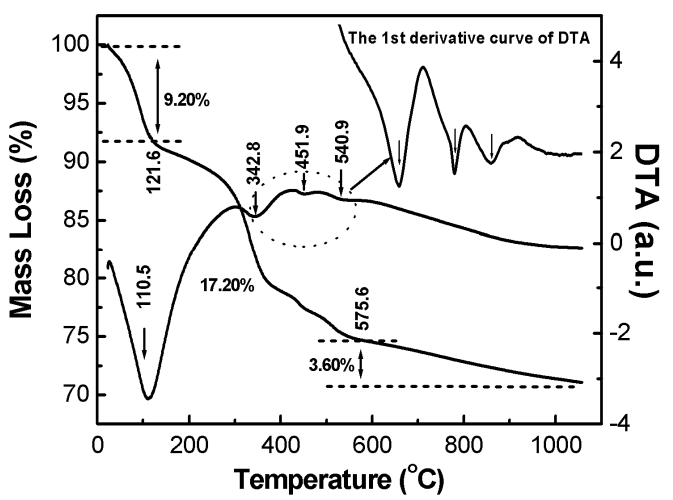


Fig. 1 DTA-TGA curves representing the decomposition of $\left(\mathrm{CH}_{3} \mathrm{COO}\right)_{2} \mathrm{Co} \cdot 4 \mathrm{H}_{2} \mathrm{O}$ contained within a CA (CA-Co-I) at a heating rate of $10^{\circ} \mathrm{C} \mathrm{min}^{-1}$ in $\mathrm{N}_{2}$.

DTA/TGA results for the decomposition of cobalt acetate contained within the CA are presented in Fig. 1. These results show $9.20 \%$ mass loss up to $\sim 121^{\circ} \mathrm{C}$ associated with water loss. A further $17.20 \%$ mass loss occurs between $121.6^{\circ} \mathrm{C}$ and $575.6^{\circ} \mathrm{C}$. This second mass loss arises due to the decomposition of the cobalt acetate into cobalt oxide via the intermediate compounds acetyl cobalt acetate and cobalt acetate hydroxide [12]. Finally, metallic Co is formed along with unfavourable Co oxides. $\mathrm{Co}_{3} \mathrm{O}_{4}$ is detected if the sample is pyrolysed in air, whereas $\mathrm{CoO}$ is found if the sample is pyrolysed under a nitrogen atmosphere [12]. In the presence of excess oxygen during pyrolysis, the $\mathrm{CoO}$ is oxidised at temperatures of $400-500^{\circ} \mathrm{C}$ forming blue cobalt (II,III) oxide $\left(\mathrm{Co}_{3} \mathrm{O}_{4}\right)$ which has the spinel structure.

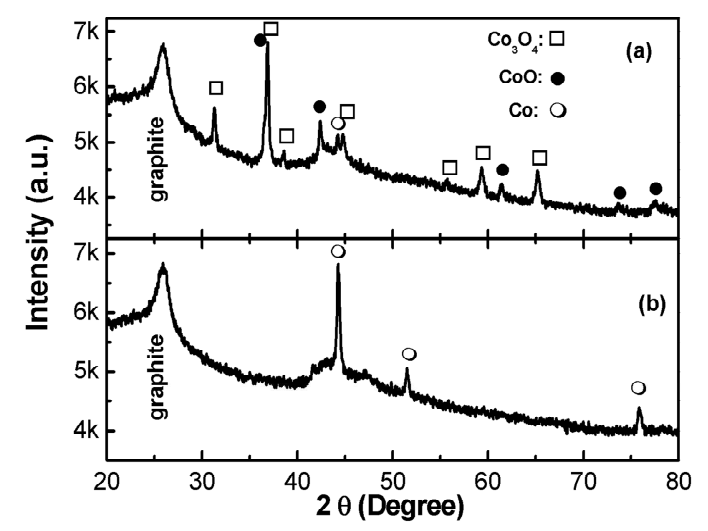

Fig. 2 X-ray diffraction patterns for (a) Co-CA- I after impregnation and decomposition of cobalt acetate in flowing nitrogen at $600^{\circ} \mathrm{C}$ for $1 \mathrm{hr}$ and (b) Co-CA-I after impregnation and decomposition of cobalt acetate in flowing nitrogen followed by reduction under flowing $\mathrm{H}_{2} / \mathrm{Ar}$ gas at $500^{\circ} \mathrm{C}$ for $2 \mathrm{hrs}$.

Fig. 2 shows X-ray diffraction patterns for (a) the cobalt doped CA (CA-Co-I) after cobalt acetate decomposition under flowing nitrogen and (b) after cobalt oxide 
reduction under flowing $\mathrm{H}_{2} / \mathrm{Ar}$. XRD showed that the samples contain both oxide phases, in addition to metallic cobalt. The diffraction peaks from $\mathrm{Co}_{3} \mathrm{O}_{4}$ and $\mathrm{CoO}$ dominate the XRD pattern for the unreduced samples (Fig. 2a). The cobalt oxides can be transformed into pure metallic cobalt by hydrogen reduction as outlined below, and as evidenced in Fig. $2 b$.

$$
\begin{aligned}
& \mathrm{Co}_{3} \mathrm{O}_{4}+4 \mathrm{H}_{2} \stackrel{\Delta}{\longrightarrow} 3 \mathrm{Co}+4 \mathrm{H}_{2} \mathrm{O}(\mathrm{g}) \uparrow \\
& \mathrm{CoO}+\mathrm{H}_{2} \stackrel{\mathrm{Co}+\mathrm{H}_{2} \mathrm{O}(\mathrm{g}) \uparrow}{\longrightarrow}
\end{aligned}
$$

2)

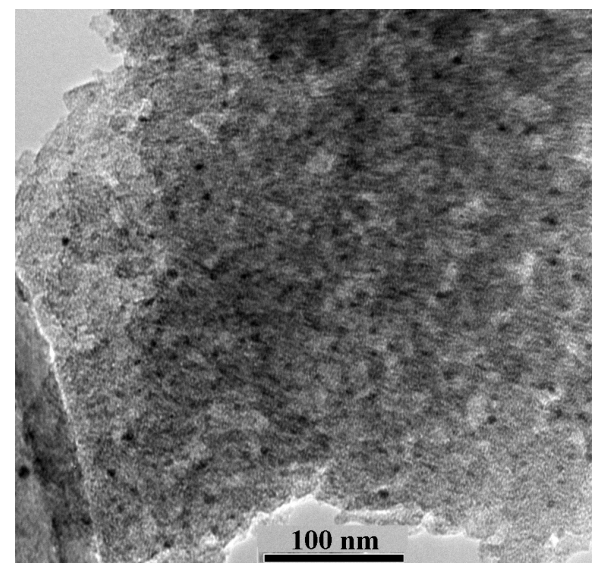

Fig. 3. TEM micrograph of a typical cobalt doped carbon aerogel sample (CA-Co-I) (scale bar in the figure is $100 \mathrm{~nm}$ ) showing well dispersed nanoscaled particles with the sizes between 2 and $8 \mathrm{~nm}$ (black dots).

A TEM micrograph of CA-Co-I is shown in Fig. 3, which displays metalcontaining particles well dispersed in the CA with particles sizes between 2 and $8 \mathrm{~nm}$. The microstructure of the pure CA and its pores can also be seen in this image as an interconnected bead structure analogous to that of typical aerogels prepared by Pekala [1]. These beads are generally several nanometers in size, and many micropores can be found with pore sizes in the range of $1-2 \mathrm{~nm}$ (determined via a pixel counting method using DigitalMicrograph software (Gatan, USA)) which agree with small angle X-ray scattering (SAXS) average pore size measurements [7]. 
Hydrogen sorption measurements were performed at both room temperature and at cryogenic temperature $\left(-196^{\circ} \mathrm{C}\right)$, with results presented in Fig. 4 . The densities of CA and Co-doped CA were used as $1.8 \mathrm{~g} \mathrm{~cm}^{-3}$ and $1.9 \mathrm{~g} \mathrm{~cm}^{-3}$ in our measurements, respectively. The mass for each sample is approximately $0.5 \mathrm{~g}$ used to evaluate the hydrogen uptake capacity. The hydrogen sorption at room temperature is much lower than at $-196^{\circ} \mathrm{C}$ because physisorption is a function of van der Waals forces. For a low pressure, the isotherm reduces to Henry's law, where the coverage of hydrogen on the carbon aerogels is proportional to the pressure, showing near-linear behaviour between hydrogen uptake and equilibrium pressure. The hydrogen adsorption for CACo-I displays a high level of hydrogen uptake $\left(4.38\right.$ wt.\%) at $-196^{\circ} \mathrm{C}$ and $4.6 \mathrm{MPa}$. The measured excess wt.\% of hydrogen is much higher than that of metal-doped CAs previously presented in the literature $(2.1 \mathrm{wt} . \%$ for $\mathrm{Co}-\mathrm{CA}$ and $2.3 \mathrm{wt} . \%$ for Ni-CA) [2]. The higher hydrogen storage capacity in the Co-CA-I is attributed to the higher surface area and large micropore volume obtained by our efficient synthesis method. However, the hydrogen uptake in CA-Co-I is approximately $10 \%$ lower than the uptake in pure CA as shown in Table 1. This phenomenon is mainly related to the decrease in micropore volume in the cobalt-doped sample, i.e. from $0.7 \mathrm{~cm}^{3} \mathrm{~g}^{-1}$ for CA to $0.6 \mathrm{~cm}^{3} \mathrm{~g}^{-1}$ for CA-Co-I. There is a large decrease $(\sim 67 \%)$ in hydrogen uptake for CA-Co-II because of the large decrease in surface area (77\%) and micropore volume (71\%). This is because the potential field generated by the micropore walls acts to enhance the binding energy between hydrogen molecules and carbon adsorbent. The decrease in the pore volume suggests that metallic particles are filling the larger pores, i.e. mesopores, which leads to a drop in the total pore volume from $1.32 \mathrm{~cm}^{3} \mathrm{~g}^{-1}$ for pure CA to $0.70 \mathrm{~cm}^{3} \mathrm{~g}^{-1}$ for the CA-Co-I. There is a small decrease in micropore volume ( drop $7 \%$ ) which may be suggestive of a degree of micropore blocking from 
the cobalt particles. It is therefore suggested that cobalt acetate molecules mainly diffuse into the bigger pores, instead of the micropores. This is consistent with the TEM results which show particle sizes larger than $2 \mathrm{~nm}$. It is worth pointing out that the micropore volume of the CA-Co-II is dramatically lower than that of the CA-Co-I, although the total pore volume is actually larger than that of CA-Co-I. The high surface area and large micropore volume associated with cobalt doped CA-Co-I suggests that samples synthesised with this method may be suitable for a variety of applications including gas sorption and catalysis due to the high degree of control during the synthesis method over surface area, pore volume, dopant species and dopant levels.

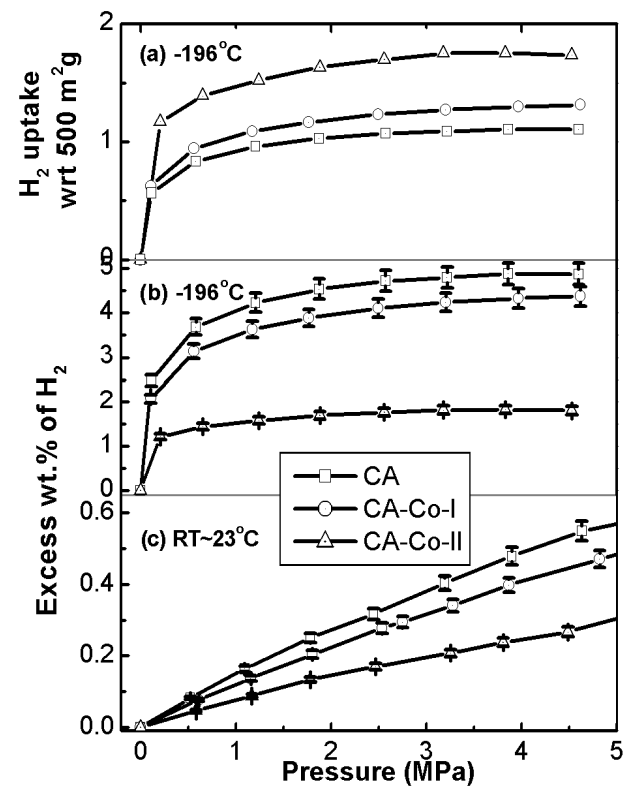

Fig. 4 (a) Hydrogen uptake per unit surface area at $-196^{\circ} \mathrm{C}$ and hydrogen sorption isotherms of CA, CA-Co-I and CA-Co-II at (b) $-196^{\circ} \mathrm{C}$ and (c) room temperature $\left(23^{\circ} \mathrm{C}\right)$.

The hydrogen uptake per unit surface area of CA (shown in Fig. 4a) is similar to that for activated carbons. i.e. $1 \mathrm{wt} . \%$ for every $500 \mathrm{~m}^{2} \mathrm{~g}^{-1}$ of surface area [2], i.e. "Chahine rule" [13]. It is worth noting that the hydrogen uptake per unit surface area of cobalt- 
Table 1 The pore structure properties as determined by nitrogen adsorption at $-196^{\circ} \mathrm{C}$.

\begin{tabular}{|c|c|c|c|c|c|}
\hline Sample & $\begin{array}{c}\text { Surface area } \\
\begin{array}{c}S_{\mathrm{BET}} \\
\left(\mathrm{m}^{2} \mathrm{~g}^{-1}\right)\end{array}\end{array}$ & $\begin{array}{c}\text { Total pore } \\
\text { volume }\left(\mathrm{cm}^{3} \mathrm{~g}^{-1}\right)\end{array}$ & $\begin{array}{c}\text { Micro-pore } \\
\text { volume } \\
\left(\mathrm{cm}^{3} \mathrm{~g}^{-1}\right)\end{array}$ & $\begin{array}{c}\text { Max. } \mathrm{H}_{2} \text { (wt.\%) } \\
\left(-196^{\circ} \mathrm{C}\right)\end{array}$ & $\begin{array}{c}\text { Max. } \mathrm{H}_{2} \\
(\mathrm{wt} . \%) \text { per } \\
500 \mathrm{~m}^{2} \mathrm{~g}^{-1}\end{array}$ \\
\hline CA & 2206 & $1.32 \pm 0.02$ & $0.70 \pm 0.02$ & $4.88(4.6 \mathrm{MPa})$ & 1.1 \\
\hline CA-Co-I & 1667 & $0.70 \pm 0.01$ & $0.60 \pm 0.01$ & $4.38(4.6 \mathrm{MPa})$ & 1.3 \\
\hline CA-Co-II & 518 & $0.92 \pm 0.01$ & $0.20 \pm 0.01$ & $1.82(3.8 \mathrm{MPa})$ & 1.8 \\
\hline
\end{tabular}

doped CAs is higher than that measured for the pure CA ( 1.1 wt.\%). CA-Co-I contains 1.3 wt. $\% \mathrm{H}_{2}$ and CA-Co-II $1.8 \mathrm{wt} . \% \mathrm{H}_{2}$ for every $500 \mathrm{~m}^{2} \mathrm{~g}^{-1}$ of surface area, respectively. The results suggest that there exist interactions between metallic particles and carbon surface which give rise to enhanced hydrogen adsorption capacity. In the case of CA-Co-II, this increased interaction strength comes with a significant penalty to the surface area and, ultimately, the total hydrogen uptake. Previous hydrogen desorption measurements on pure CA do not show hysteresis. Additionally, Kabbour et al. [2] have shown that there is no hysteresis associated with hydrogen desorption in their cobalt and Ni doped CAs. As such we expect little or no hysteresis to be associated with hydrogen desorption from CA-Co-I. The method we have used to dope carbon aerogels is actually dependent on the carbon aerogels having mesoporosity. Activated carbons are typically almost completely microporous (pore size $<2 \mathrm{~nm}$ ) [14]. Hence, the size of solvated cobalt acetate tetrahydrate molecules is typically too large to fit into most of these pores $(\sim 13.5 \AA$ by our calculations) and any cobalt particles formed would be external to the pore structure. Having the cobalt particles within the pore structure will take advantage of the high surface area for catalytic applications and gas adsorption. Producing cobalt within the smaller pores of activated carbon would be dependent on finding a reagent that has a smaller solvation size yet can still be reduced back to metallic cobalt. On the basis of our results, the flexibility of chemistry synthesis approaches allows for the incorporation of metal dopants into a porous carbon matrix to enhance hydrogen 
uptake in Co doped CA with respect to unit surface area or micropore volume. However, whether the adsorption mechanism is due to surface heterogeneity, to the interaction among adsorbed molecules, or to the interaction between hydrogen molecules and metallic particles is yet to be determined from our measurements. Research is now in progress to clarify the role of metallic particles during hydrogen adsorption, e.g. the effects of metal dopants on the heat of adsorption.

\section{Conclusions}

In this communication, metal doped CA with metallic cobalt particles between 2 and $8 \mathrm{~nm}$ were prepared by a wet-thin layer coating method of cobalt acetate followed by decomposition at $600^{\circ} \mathrm{C}$ under a nitrogen atmosphere and reducing at $500^{\circ} \mathrm{C}$ under a hydrogen atmosphere. After synthesis was complete a high hydrogen uptake was obtained ( 4.38 wt.\%) at 4.6 $\mathrm{MPa}$ and $-196^{\circ} \mathrm{C}$, which is greater than twice the adsorption measured for Co-doped CA derived from an ion-exchange method. A hydrogen uptake of $0.47 \mathrm{wt} . \%$ can be achieved at $4.8 \mathrm{MPa}$ and $23^{\circ} \mathrm{C}$. The results demonstrate that this new method of metal-doped CA synthesis results in high surface area samples with large micropore volumes that are beneficial to hydrogen sorption. An enhanced hydrogen uptake with $15 \%$ increase from $1.1 \mathrm{wt} . \%$ for every $500 \mathrm{~m}^{2} \mathrm{~g}^{-1}$ of surface area can be obtained in the metallic doped CA with respect to unit surface area.

\section{Acknowledgements}

This research was supported by the CSIRO Energy Transformed Flagship National Hydrogen Material Alliance (NHMA). C.E.B. acknowledges the financial support of the Australian Research Council LEIF grant LE0775551. 


\section{References}

[1] Pekala RW. Organic aerogels from the polycondensation of resorcinol with formaldehyde. J Mater Sci 1989;24:3221-7.

[2] Kabbour H, Baumann TF, Satcher Jr JH, Saulnier A, Ahn CC. Toward new candidates for hydrogen storage: high-surface-area carbon aerogels. Chem of Mater 2006;18:6085-7.

[3] Yürüm Y, Taralp A, Veziroglu TN, Storage of hydrogen in nanostructured carbon materials. Int J Hydrogen Energy 2009;34:3784-3798.

[4] Tian HY, Buckley CE, Wang SB, Zhou MF, Enhanced hydrogen storage capacity in carbon aerogels treated with KOH. Carbon 2009; 47:2128-30.

[5] Fu RW, Dresselhaus MS, Dresselhaus G, Zheng B, Liu J, Satcher JH, Baumann TF, The growth of carbon nanostructures on cobalt-doped carbon aerogels. J NonCryst. Solids 2003;318:223-32.

[6] Fu RW, Baumann TF, Cronin S, Dresselhaus G, Dresselhaus MS, Satcher Jr JH, Formation of graphitic structures in cobalt- and nickel-doped carbon aerogels, Langmuir 2005;21:2647-51.

[7] Tian HY, Buckley CE, Mulè S, Paskevicius M, Dhal BB. Preparation, microstructure and hydrogen sorption properties of nanoporous carbon aerogels under ambient drying. Nanotechnology 2008;19:475605 1-7.

[8] Basta AH, Fierro V, El-Saied H, Celzard A, 2-Steps KOH activation of rice straw: An efficient method for preparing high-performance activated carbons. Bioresource Technology 2009;100:3941-3947. 
[9] Hemmes H, Driessen A, Griessen R, Thermodynamic properties of hydrogen at pressures up to 1 Mbar and temperatures between 100 and 1000 K. J Phys. C: Solid State Phys 1986;19:3571-85

[10] McLennan KG, Gray EM, An equation of state of deuterium gas to 1000 bar.

Meas. Sci. Technol. 2004;15:211-5.

[11] Lemmon EW, McLinden MO, Friend DG. Thermophysical properties of fluid systems. In: Linstrom PJ, Mallard WG, editors. NIST chemistry WebBook. NIST Standard Reference Database Number 69. National Institute of Standards and Technology, Gaithersburg, MD, 2005. 20899. Available from: http://webbook.nist.gov.

[12] Mohamed MA, Halawy SA, and Ebrahim MM, The non-isothermal decomposition of cobalt acetate tetrahydrate: a kinetic and thermodynamic study. $\mathbf{J}$ Thermal Analysis 1994;41:387-404.

[13] Panella B, Hirscher M, Roth S. Hydrogen adsorption in different carbon nanostructures. Carbon 2005;43:2209-2214.

[14] Fierro V, Zhao W, Izquierdo MT, Aylon E, Celzard A, Adsorption and compression contributions to hydrogen storage in activated anthracites. Int $\mathbf{J}$ Hydrogen Energy 2010;35:9038-9045. 